\title{
Long Non-Coding RNA in Esophageal Cancer: A Review of Research Progress
}

\author{
Chenbo Yang ${ }^{1,2}$ and Kuisheng Chen ${ }^{1,2 *}$ \\ ${ }^{1}$ Department of Pathology, The First Affiliated Hospital of Zhengzhou University, Zhengzhou, China, ${ }^{2}$ Henan Key Laboratory of \\ Tumor Pathology, Zhengzhou University, Zhengzhou, China
}

In recent years, there has been significant progress in the diagnosis and treatment of esophageal cancer. However, owing to the lack of early diagnosis strategies and treatment targets, the prognosis of patients with esophageal cancer remains unsatisfactory. There is an urgent need to identify novel biomarkers and treatment targets for esophageal cancer. With the development of genomics, long-chain non-coding RNAs (LncRNAs), which were once considered transcriptional "noise," are being identified and characterized rapidly in large numbers. Recent research shows that LncRNAs are closely related to a series of steps in tumor development and play an important regulatory role in DNA replication, transcription, and post-transcriptional regulation. The abnormal expression of LncRNAs leads to tumor cell proliferation, migration, invasion, and treatment resistance. This review focuses on the latest progress in research on the abnormal expression and functional mechanisms of LncRNAs in esophageal cancer. Further, it discusses the potential applications of these findings towards achieving an early diagnosis, improving treatment efficacy, and evaluating the prognosis of esophageal cancer.

Keywords: biomarkers, long non-coding RNA, molecular mechanism, esophageal cancer (EC), genomics

\section{INTRODUCTION}

OPEN ACCESS

Edited by:

József Tímár.

Semmelweis University, Hungary

${ }^{*}$ Correspondence: Kuisheng Chen chenksh2002@163.com

Received: 20 October 2021 Accepted: 26 January 2022 Published: 15 February 2022

Citation: Yang $C$ and Chen $K$ (2022) Long NonCoding RNA in Esophageal Cancer: A

Review of Research Progress. Pathol. Oncol. Res. 28:1610140. doi: 10.3389/pore.2022.1610140
Esophageal cancer is one of the most common malignant tumors in the world, with the sevenhighest incidence and sixth-highest mortality rate among all malignant tumors worldwide (1). Esophageal cancer is highly aggressive, which often leads to a poor prognosis. According to global cancer statistics, 1 of every 20 cancer-related deaths in 2018 was due to esophageal cancer $(2,3)$. There are two primary histological subtypes of esophageal cancer: esophageal squamous cell carcinoma (ESCC) and esophageal adenocarcinoma (EAC). ESCC is more common between the two, accounting for $90 \%$ of all esophageal cancer cases in China. With the advancement of diagnostic technology, including novel techniques such as narrow-band imaging with magnifying endoscopy and positron emission tomography, the accuracy of esophageal cancer diagnosis has improved greatly. In addition, advances in treatment methods, such as endoscopic surgery and neoadjuvant chemotherapy, have also significantly improved treatment efficacy. However, strategies for early diagnosis are still lacking, and most cases of esophageal cancer are diagnosed in the middle or late stages, rendering surgical treatment ineffective and leading to very poor 5-year survival rates (4). Therefore, novel biomarkers and therapeutic targets for esophageal cancer are urgently required to motivate the further development of tumor-targeted drugs and early diagnosis strategies. 


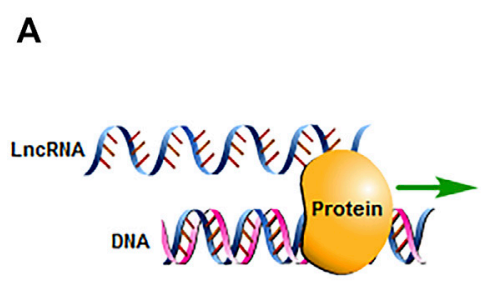

C

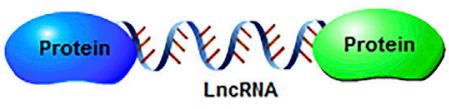

B

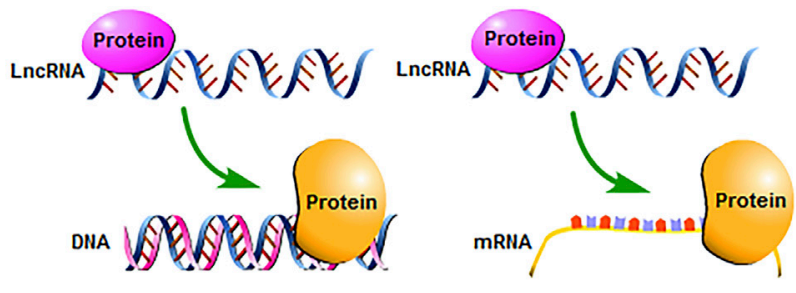

D
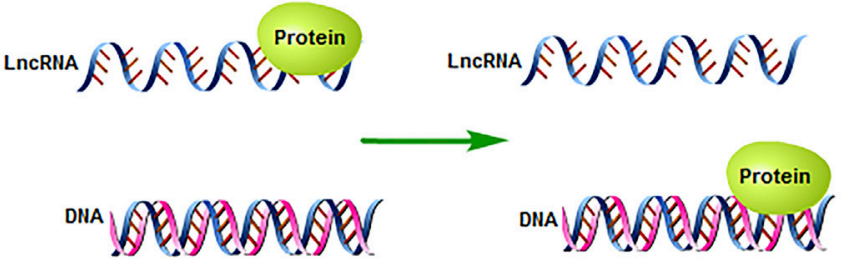

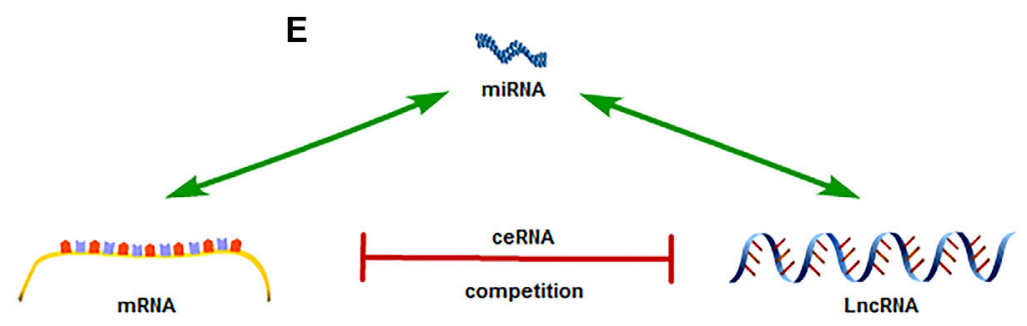

FIGURE 1 | Mechanism underlying LncRNA action in tumors. (A) LncRNAs directly act as signaling molecule or bind to proteins to regulate downstream gene expression. (B) LncRNAs bind to functional proteins and affect the regulation of DNA and mRNA by protein molecules. (C) $L$ ncRNAs act as a "scaffold" to enable related macromolecular complexes to work together in the target area. (D) LncRNAs recruit functional proteins and locate and bind to the target area to exert any action. (E) LncRNA can be used as the "molecular sponge" of miRNA. In the form of base complementary pairing, LncRNA and target gene competitively bind miRNA, thereby reducing the silencing effect of miRNA on target gene, and further affecting the regulation of miRNA on downstream target gene.

The Human Genome Project has shown that the human genome contains about 20,000 protein-coding genes, which only account for $1.5 \%$ of all genes (5). Transcription products that are greater than 200 nucleotides in length and do not participate in protein expression are called long-chain noncoding RNAs (LncRNAs). Owing to a lack of meaningful open reading frames and protein -encoding functions, LncRNAs were once considered transcriptional "noise." However, with the subsequent development of molecular biology, it was gradually discovered that LncRNAs regulate gene expression at different levels (epigenetic, transcriptional, and post-transcriptional) and participate in processes such as cell growth and apoptosis, protein activity regulation, and variable splicing $(6,7)$. For example, LncRNA-DILC binds to the IL- 6 promoter, changes the gene transcription of the binding region, and inhibits the transcription of IL-6 (8) (Figure 1A); LncRNA-H19 can inhibit S-adenosine homocysteine hydrolase and increase the level of S-adenosine homocysteine. Thereby, the inhibitory effect on S-adenosylmethionine-dependent methyltransferase is enhanced, and it induces a wide range of methylation changes in the whole genome (9) (Figure 1B); LncRNAHOTAIR can be used as a molecular scaffold to bind to histone modification complexes, combine to perform specific functions in different regions, and mediate histone methylation and demethylation (10) (Figure 1C); LncRNA$\mathrm{HEIH}$ binds to histone methyltransferase EZH2, recruits EZH2 to the promoter region of the target gene, induces methylation of the promoter region of the target gene, thereby inhibiting the expression of the target gene (11) (Figure 1D); LncRNA can be used as the "molecular sponge" of miRNA. In the form of base complementary pairing, LncRNA and target gene competitively bind miRNA, thereby reducing the silencing effect of miRNA on target gene, and further affecting the regulation of miRNA on downstream target gene (Figure 1E). Studies have also found a link between the abnormal expression and dysfunction of LncRNAs and human diseases, especially malignant tumors. Therefore, LncRNAs are considered to be of great significance in tumor diagnosis, treatment, and prognostication (12).

This article reviews the latest research on the functional role and molecular mechanisms of LncRNA in esophageal cancer. Further, it discusses the significance of LncRNA in the diagnosis, treatment, and prognosis of esophageal cancer and describes the potential applications of LncRNAs as biomarkers and therapeutic targets for esophageal cancer. 


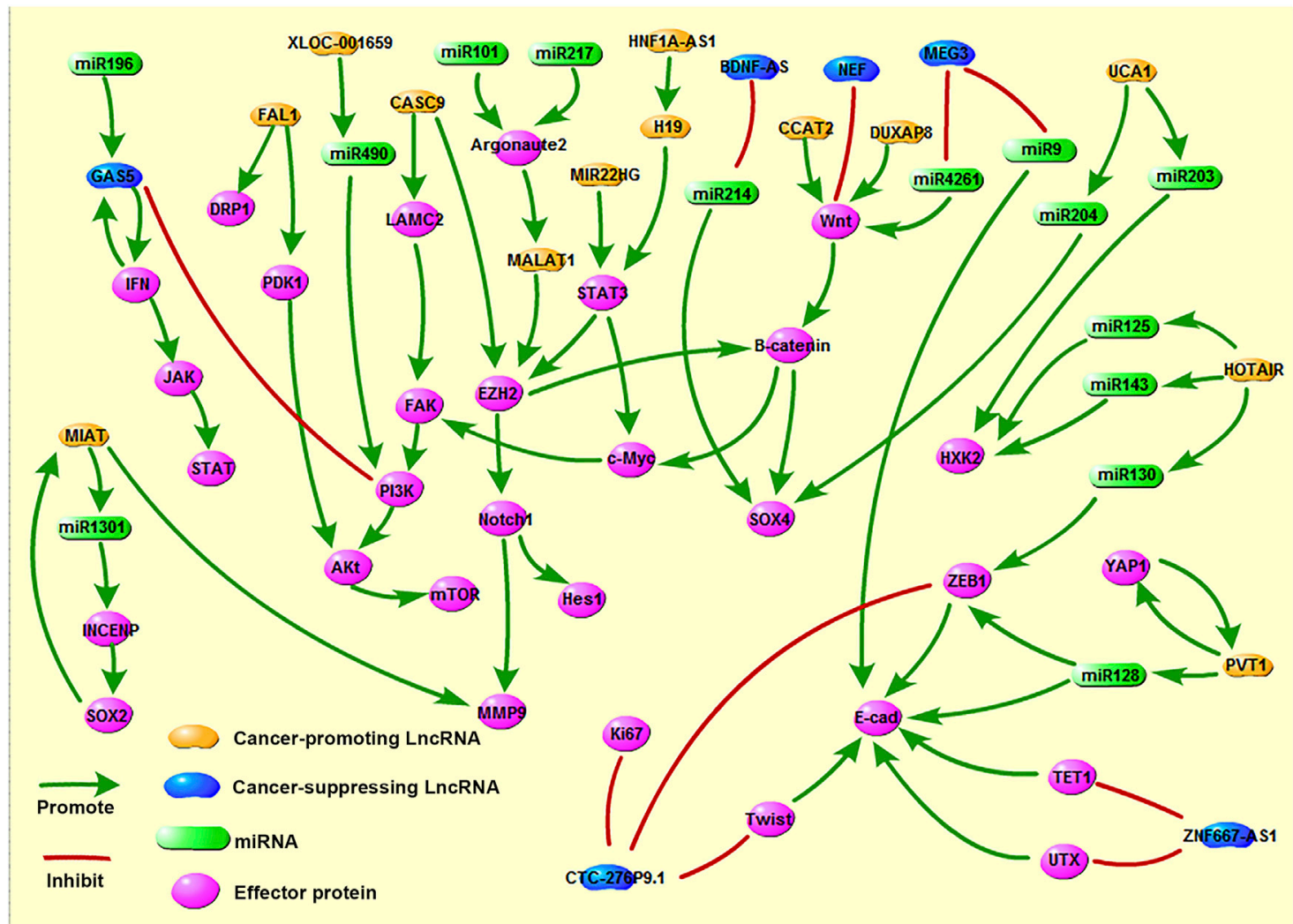

FIGURE 2 | Molecular network of common LncRNAs associated with esophageal cancer.

\section{LONG-CHAIN NON-CODING RNAS PROMOTE THE OCCURRENCE AND DEVELOPMENT OF ESOPHAGEAL CANCER}

A large number of studies have pointed out that most LncRNAs act as oncogenes in the occurrence and development of tumors (Figure 2). These LncRNAs are highly expressed in tumor tissues; promote tumor cell proliferation, migration, and invasion through various mechanisms; regulate the cell cycle; and inhibit cell apoptosis. Screening out LncRNAs with esophageal cancer-promoting effects can further elucidate the occurrence and development of tumors (Table 1). Such information can provide tools that allow the suppression of tumor growth and metastasis via gene knock-out or silencing and thereby improve prognosis (13).

Metastasis-related lung adenocarcinoma transcript 1 (MALAT1) is located on human chromosome $11 \mathrm{q} 13$ and was first discovered in lung adenocarcinoma tissue. This gene is a marker for the metastasis and prognosis of non-small cell lung cancer (14). MALAT1 is highly expressed in a variety of tumor tissues and is involved in tumor regulation. Hu et al. (15) showed that MALAT1 is highly expressed in advanced esophageal cancer tissues and does not play a role in the initial stage of the tumor. Silencing the MALAT1 gene activates the ATM-Chk2 pathway, arrests the cell cycle in the G2/M phase, and increases the rate of apoptosis. In ESCC, MALAT1 exerts cancer-promoting effects via the dephosphorylation of the ATM, Chk2 protein. Chen et al. (16) found that in ESCC, MALAT1 regulates the expression of Hes1 and MMP9 through the EZH2-Notch1 signaling pathway, which affects the proliferation, migration, and invasion of ESCC cells. Several LncRNAs have been found to act as ceRNAs by sponging miRNAs to reduce their inhibitory effect on their target proteincoding mRNAs. However, miRNA can also regulate the expression of LncRNA through counteraction. In ESCC, miR101 and miR-217 act as tumor suppressor genes and downregulate the expression of MALAT1 through an Argonaute2mediated pathway, thereby inhibiting cell migration and invasion (17).

H19 is a type of commonly studied cancer-promoting LncRNA (18). Chen et al. (19) experimentally found that H19 is highly expressed in esophageal cancer cell lines. A nude mouse xenograft model showed that $H 19$ knockout significantly reduced the size and weight of xenograft tumors and the protein expression of STAT3, SOX4, EZH2, and $\beta$-catenin. This demonstrates that $H 19$ mediates the malignant progression of esophageal cancer via the STAT3/EZH2/ $\beta$-catenin axis in vivo and in vitro. HNF1A-AS1 is upstream of H19. When HNF1A-AS1 is inhibited, $H 19$ also gets inhibited, and tumor cell survival and metastasis decrease. Genes related to nucleosome and chromatin assembly in the G1/S phase of the cell cycle are also significantly 
TABLE 1 | Mechanisms of common IncRNAs in regulating the progression of esophageal cancer.

\begin{tabular}{|c|c|c|c|c|}
\hline LncRNA & Histological type & Effects on tumor & Mechanism & References \\
\hline \multirow[t]{3}{*}{ MALAT1 } & ESCC & Promote & Regulates ATM-CHK2 pathway & $(15)$ \\
\hline & ESCC & Promote & Regulates EZH2-Notch1 pathway mediates Hes1 and MMP9 proteins & $(16)$ \\
\hline & ESCC & Promote & Regulated by miR-101 and miR-217 & $(17)$ \\
\hline \multirow[t]{2}{*}{$\mathrm{H} 19$} & Esophageal cancer & Promote & Regulates STAT3/EZH2/ $\beta$-catenin axis & (19) \\
\hline & EAC & Promote & Regulated by LncRNA-HNF1A-AS1 & (20) \\
\hline \multirow[t]{3}{*}{ UCA1 } & ESCC & Promote & Adsorbs miR-498 as ceRNA to regulate ZEB2 expression & $(21)$ \\
\hline & ESCC & Promote & Adsorbs miR-204 as ceRNA to regulate SOX4 expression & $(22)$ \\
\hline & ESCC & Promote & Adsorbs miR-203 as ceRNA to regulate HK2 expression & (23) \\
\hline \multirow[t]{2}{*}{ MIAT } & ESCC & Promote & Regulates the expression of cyclin D3, CDK2, MMP2 and MMP9 proteins & (24) \\
\hline & ESCC & Promote & Adsorbs miR-1301-3p as ceRNA to regulate INCENP expression & (25) \\
\hline \multirow[t]{2}{*}{ PVT1 } & EAC & Promote & Regulates the expression of LATS1 and YAP1 proteins & (26) \\
\hline & ESCC & Promote & Regulates miR-128/ZEB1/E-cadherin axis & $(27)$ \\
\hline \multirow[t]{2}{*}{ CCAT2 } & ESCC & Promote & Regulates $\beta$-catenin/WISP1 axis & (29) \\
\hline & ESCC & Promote & Regulates Wnt/ $\beta$-catenin pathway & (30) \\
\hline \multirow[t]{2}{*}{ CASC9 } & ESCC & Promote & LAMC2-mediated FAK-PI3K/Akt pathway, binds to CBP and modifies histone acetylation & (31) \\
\hline & ESCC & Promote & Mediates the recruitment of $\mathrm{EZH} 2$ and $\mathrm{H} 3 \mathrm{~K} 27 \mathrm{me} 3$, regulates the expression of PDCD4 protein & (32) \\
\hline \multirow[t]{2}{*}{ FAL1 } & ESCC & Promote & Regulates the expression of DRP1 protein and mitochondrial dynamics & (34) \\
\hline & ESCC & Promote & Regulates the expression of PDK1 protein, activates the AKt signaling pathway & (35) \\
\hline \multirow[t]{2}{*}{ HOTAIR } & ESCC & Promote & Adsorbs miR-125/miR-143 as ceRNA to regulate HK2 expression & $(37)$ \\
\hline & ESCC & Promote & Adsorbs miR-130a-5p as ceRNA to regulate ZEB1 expression & $(38)$ \\
\hline $\mathrm{MIR} 22 \mathrm{HG}$ & EAC & Promote & Regulates STAT3/c-Myc/p-FAK pathway & (39) \\
\hline XLOC-001659 & ESCC & Promote & Adsorbs miR-490-5p as ceRNA to regulate PIK3CA expression & $(40)$ \\
\hline DUXAP8 & ESCC & Promote & Regulates Wnt/ $\beta$-catenin pathway & $(41)$ \\
\hline VESTAR & ESCC & Promote & Binds and stabilizes VEGFC mRNA & $(42)$ \\
\hline PCAT1 & ESCC & Promote & Regulated by miR-326 & $(77)$ \\
\hline \multirow[t]{3}{*}{ GAS5 } & ESCC & Inhibit & Regulates PI3K/AKt/mTOR pathway & $(44)$ \\
\hline & ESCC & Inhibit & Regulates IFN/JAK/STAT axis & $(45)$ \\
\hline & ESCC & Inhibit & Regulated by miR-196a & $(46)$ \\
\hline NEF & ESCC & Inhibit & Regulates Wnt/ $\beta$-catenin pathway & $(48)$ \\
\hline BDNF-AS & EAC & Inhibit & Adsorbs miR-214 as ceRNA & $(50)$ \\
\hline \multirow[t]{2}{*}{ MEG3 } & ESCC & Inhibit & Adsorbs miR-204 as ceRNA to regulate E-cadherin and FOXO1 expression & $(51)$ \\
\hline & ESCC & Inhibit & Regulates miR-4261/Wnt/ $\beta$-catenin axis & $(52)$ \\
\hline $\begin{array}{l}\text { ADAMTS9- } \\
\text { AS2 }\end{array}$ & EAC & Inhibit & Regulates the expression of $\mathrm{CDH} 3$ proteins & $(54)$ \\
\hline ZNF667-AS1 & ESCC & Inhibit & Regulates the expression of E-cadherin,and ZNF667 proteins & $(55)$ \\
\hline CTC-276P9.1 & ESCC & Inhibit & Regulates the expression of Ki67, PCNA, Twist1 and ZEB1 proteins & $(56)$ \\
\hline NBAT-1 & ESCC & Inhibit & Regulates the expression of PKM2 proteins & $(57)$ \\
\hline SEMA3B-AS1 & ESCC & Inhibit & Regulates the expression of SEMA3B proteins & $(58)$ \\
\hline uc061hsf.1 & ESCC & Inhibit & Regulated by p53 & $(61)$ \\
\hline
\end{tabular}

affected. The molecular mechanisms via which HNF1A-AS1 regulates esophageal cancer include the regulation of $\mathrm{H} 19$ expression and effects on the nucleosome and chromatin assembly pathways (20).

UCA1 has multiple molecular functions in esophageal cancer. In esophageal cancer cells, UCA1 uses ceRNA to adsorb miR-498, causing changes in downstream ZEB2 expression and exerting cancer-promoting effects (21). UCA1 can also act as a ceRNA to regulate the expression of the target gene SOX4. UCA1 also binds to miR-204 to inhibit the degradation of SOX4, thereby promoting the invasion and metastasis of tumor cells (22). Studies have found that UCA1 also promotes the expression of HXK2 by interacting with miR-203, thereby facilitating the aerobic glycolysis of tumor tissues and enhancing the Warburg effect to promote tumor cell proliferation and metastasis (23).

Myocardial infarction-associated transcript (MIAT), located on human chromosome 22 (19), was originally discovered as a gene associated with the risk of myocardial infarction. Zhang et al. (24) knocked out MIAT and found that the survival rate of esophageal cancer cells was reduced, the expression of cyclin 
D3 and CDK2 was reduced, and cell cycle arrest occurred in the G1 phase, leading to the inhibition of cell proliferation. Further, the levels of MMP2 and MMP9 were significantly downregulated, and metastasis was inhibited. MIAT can act as a ceRNA to mediate the up-regulation of inner centromere protein (INCENP) by miR-1301-3p, forming a feedback pathway with SOX2 (MIAT-SOX2) and promoting the proliferation, migration, and invasion of ESCC cells (25).

The plasmacytoma variant translocation 1 (PVT1) gene, located on human chromosome 8q24, was the first LncRNA found in human cancer. After knocking out PVT1 in EAC cells, the phosphorylation of LATS1 and YAP1 increased and their protein function was lost, leading to the inhibition of tumor proliferation and invasion. YAP1 knock-out also significantly inhibited the expression of PVT1; if YAP1 was overexpressed, the level of PVT1 increased significantly. This indicated that LATS1 and YAP1 are downstream effectors of PVT1, and YAP1 has a positive feedback effect on PVT1 (26). Hu et al. (27) suggested that PVT1 promotes the downstream expression of ZEB1 and E-cadherin via miR-128, promoting ESCC progression.

Colon cancer-associated transcript 2 (CCAT2), which is located on chromosome $8 \mathrm{q} 24$ and expressed in microsatellite -stable colon cancer, was first identified as a single nucleotide polymorphism region (28). CCAT2 silencing was found to downregulate $\beta$-catenin and Wnt-mediated secreted protein (WISP1) expression and significantly weaken the proliferation and migration ability of tumor cells (29). Treatment of esophageal cancer cells with a Wnt inhibitor (FH535) recapitulated the effects of CCAT2 inhibition, indicating that CCAT2 exerts cancer-promoting effects through the $\mathrm{Wnt} / \beta$-catenin pathway (30).

As a carcinogenic LncRNA, CASC9 is mostly present in squamous epithelial tumors and was first discovered in ESCC (13). Liang et al. (31) suggested that CASC9 promotes esophageal cancer metastasis through the FAK-PI3K/Akt pathway via LAMC2 and that CASC9 stimulates the expression of LAMC2 through CBP-mediated histone acetylation to promote the invasion and metastasis of esophageal cancer. Silencing CASC9 inhibits the growth of ESCC cells and transplanted tumors in nude mice. CASC9 mediates the recruitment of $\mathrm{EZH} 2$ and $\mathrm{H} 3 \mathrm{~K} 27 \mathrm{me} 3$ to the promoter region and regulates the expression of PDCD4, thereby exerting cancer-promoting effects (32).

FAL1 is a LncRNA that has strongly been linked to malignant tumors in recent years. It is locally amplified on chromosome 1 and has a carcinogenic effect (33). Liu et al. (34) discovered a new molecular mechanism for the regulation of esophageal cancer progression. FAL1 enhances the proliferation of ESCC cells by regulating the expression of mitochondrial division protein (DRP1) and mitochondrial dynamics. The down-regulation of FAL1 can promote the expression of DRP1, and intracellular mitochondrial division is related to DRP1-mediated mitochondrial dysfunction. Therefore, FAL1 silencing leads to mitochondrial dysfunction and promotes apoptosis by inhibiting mitochondrial respiration and ATP production. FAL1 can also target PDK1 expression and activate the AKt signaling pathway, thereby promoting the proliferation of ESCC cells (35).
HOX transcribed antisense intergenic RNA (HOTAIR) is located in the HOXC gene cluster and has a length of 685 nucleotides. It was originally discovered as a regulator of tumor invasion and metastasis in breast cancer. At present, HOTAIR is a well-known LncRNA (36). Ma et al. (37) found that HOTAIR can effectively act as a "molecular sponge" for miR$125 / \mathrm{miR}-143$, negatively regulate the expression of miR-125/ miR-143, promote the expression of $\mathrm{HK} 2$, and ultimately promote the occurrence and development of ESCC. Wang et al. (38) found that HOTAIR acts as a ceRNA after binding to $\mathrm{miR}-130 \mathrm{a}-5 \mathrm{p}$, regulates the expression of the downstream protein ZEB1, and promotes the epithelial-mesenchymal transition (EMT) in ESCC.

Some carcinogenic LncRNAs have been identified for the first time in esophageal cancer in recent years. Current research on these newly discovered cancer-promoting LncRNAs is not comprehensive and detailed, and further research is thus warranted. Su et al. (39) found that MIR22HG in EAC cells promotes tumor cell proliferation, anti-apoptotic effects, migration, and invasion through the STAT3/c-Myc/p-FAK pathway. Li et al. (40) used a LncRNA microarray analysis and found that the expression of XLOC-001659 in esophageal cancer tissues is 20.9 times higher than that in normal esophageal tissues. XLOC-001659 uses ceRNA to bind to miR-490-5p and promotes the occurrence and development of ESCC via the miR490-5p/PIK3CA axis. Xu et al. (41) postulated that DUXAP8 regulates the expression of cyclin D1 and $\mathrm{c}-\mathrm{Myc}$ through the $\mathrm{Wnt} / \beta$-catenin pathway and thereby promotes the proliferation and invasion of esophageal cancer cells. In addition to regulating downstream genes through ceRNA action, some LncRNAs also act as gene -stabilizing factors to regulate target genes, affecting protein expression and promoting cancer phenotypes. VESTAR, a stable VEGF-C LncRNA, can directly bind VEGF-C mRNA and maintain the stability of the mRNA structure. VEGF-C is known to be the most effective pro-lymphangiogenic factor. The high expression of VESTAR in ESCC tissue indicates that it may be involved in ESCC lymph node metastasis. $\mathrm{HuR}$ is a positive regulator of VEGF-C stability. VESTAR interacts with HuR to promote the binding of HuR to VEGF-C mRNA. Therefore, VESTAR-mediated VEGF-C mRNA stability may be an important contributor to lymph node metastasis in ESCC and could be a novel target for the diagnosis and treatment of tumor metastasis (42).

\section{LONG-CHAIN NON-CODING RNAS INHIBIT THE OCCURRENCE AND DEVELOPMENT OF ESOPHAGEAL CANCER}

Although most LncRNAs are known to have cancer-promoting effects, some LncRNAs that act as tumor-suppressor genes have also been identified (Figure 2). Tumor suppressor LncRNAs inhibit tumor proliferation and migration through a variety of molecular mechanisms and also promote tumor cell apoptosis (Table 1). However, there are high levels of epigenetic regulation in tumor tissues, and the promoter region of most tumor suppressor LncRNAs are methylated. Consequently, their 
expression is inhibited and their tumor-suppressing action fails. Therefore, the demethylation of these promoters and upregulation of gene expression could be a potential strategy for cancer treatment.

GAS5 is a typical tumor suppressor LncRNA; it has been found to have a significant ability to inhibit tumor cell proliferation and promote apoptosis in lung, breast, and colorectal cancer (43). Wang et al. (44) found that GAS5 overexpression in esophageal cancer cells significantly down-regulates the expression of PI3K and the phosphorylation levels of Akt and mTOR. The use of PI3K activators was found to reverse the inhibitory effect of GAS5 overexpression on tumor proliferation and migration, suggesting that GAS5 inhibits tumors by inactivating the PI3K/AKt/mTOR pathway. Huang et al. (45) discovered another anti-tumor effect of GAS5. They found a feedback pathway between IFN and GAS5, which exerts an active anti-tumor effect. When ESCC cells are cultured and treated with IFNs, the JAK-STAT signaling pathway is activated, and the expression of GAS5 increases. This increase in GAS5 expression in ESCC cells also promotes the interferon response in tumor cells and up-regulates the levels of IFN. GAS5 is a positive regulator of IFN. The low expression of most tumor suppressor LncRNAs in tumor tissues is often a result of promoter methylation. However, the low expression of GAS5 in ESCC may be a result of the effects of miR-196a. miR-196a binds to GAS5 and down-regulates GAS5 levels, reducing its antitumor effect considerably (46). miR-196a is an important oncogene. The silencing of miR-196a expression would not only inhibit its cancer-promoting effect but also increase the levels of GAS5, a potential target for ESCC treatment.

$N E F$ is a new type of tumor suppressor LncRNA that has been discovered in hepatocellular carcinoma in recent years. NEF inhibits $\mathrm{Wnt} / \beta$-catenin pathway to achieve tumor suppressor effect in hepatocellular carcinoma (47). Zhang et al. (48) also found that $N E F$ overexpression can reduce the expression of $\mathrm{Wnt} / \beta$-catenin pathway -related proteins in ESCC cells and thereby inhibit tumor cell proliferation, migration, and invasion. In esophageal cancer, NEF also participates in the inhibition of esophageal cancer by regulating the $\mathrm{Wnt} / \beta$-catenin pathway.

Brain-derived neurotrophic factor antisense LncRNA (BDNF$A S)$ is a natural non-coding antisense RNA of the neural transcription factor BDNF, which is important for the nervous system (49). miR-214 is a crucial cancer-promoting factor. $B D N F-A S$, as the "molecular sponge" of miR-214, inhibits the proliferation, migration, and invasion of esophageal cancer cells and inhibits the EMT (50).

Maternally expressed gene 3 (MEG3) is located at $14 \mathrm{q} 32$. Studies have found that chromosomal abnormalities in this region are closely related to the occurrence and development of tumors. Dong et al. (51) found that MEG3 acts as a ceRNA and competitively binds to miR-9 and regulates the expression of E-cadherin and FOXO1, consequently interfering with the proliferation and invasion of esophageal cancer cells. Huang et al. (52) used in vivo and in vitro experiments to show that MEG3 can target miR-4261 and thereby block the Wnt/ $\beta$ catenin signaling pathway, inhibiting tumor occurrence and development.

In recent years, researchers have found that some tumor suppressor LncRNAs in esophageal cancer directly act on downstream target proteins to regulate their expression and function. ADAMTS9-AS2 is the antisense transcript of ADAMTS9, which acts as a tumor suppressor gene in gliomas and inhibits tumor angiogenesis. Further, it is also known to inhibit tumor formation in esophageal and nasopharyngeal cancer (53). ADAMTS9-AS2 recruits DNMT1/DNMT3 to the promoter region of $\mathrm{CDH} 3$, resulting in $\mathrm{CpG}$ island hypomethylation and inhibiting the expression of $\mathrm{CDH} 3$, thereby inhibiting cancer cell function (54). ZNF667-AS1 is located in the nucleus of esophageal cancer cells. ZNF667-AS1 can recruit TET1 to interact with ZNF667 and E-cadherin and hydrolyze $5^{\prime}$-MC to $5^{\prime}$-HMC to further activate its expression. Moreover, ZNF667-AS1 also changes the H3K27 methylation status in the promoter region of ZNF667 and the E-cadherin gene by interacting with UTX, thereby regulating the transcription and expression of these genes and exerting anti-tumor effects (55). CTC-276P9.1 is also an important tumor suppressor that can inhibit the proliferation and EMT of esophageal cancer cells by directly regulating the expression of downstream proteins. Guo et al. (56) found that CTC-276P9.1 overexpression in esophageal cancer cells can significantly reduce the expression of Ki67 and PCNA and inhibit cell proliferation. Furthermore, CTC-276P9.1 regulates the expression of E-cadherin and Vimentin by regulating the transcription of Twist1 and ZEB1 and ultimately affects the EMT of esophageal cancer cells. Zhao et al. (57) found that the overexpression of NBAT-1 significantly down-regulates PKM2, a key metabolic enzyme, whose disruption affects the function of tumor cells. Therefore, it has been speculated that the tumor-suppressing effect of NBAT-1 on esophageal cancer depends on PKM2-mediated tumor glycolysis. Semaphore 3B (SEMA3B) is a tumor suppressor gene located on human chromosome $3 \mathrm{q} 21.3$. Its antisense long noncoding RNA (SEMA3B-AS1) is often inactivated in ESCC and loses its anti-tumor effect. $\mathrm{CpG}$ dinucleotide hypermethylation in the promoter region of $S E M A 3 B-A S 1$ does not affect the transcription of SEMA3B but inhibits the expression of the SEMA3B protein, indicating that $S E M A 3 B-A S 1$ may regulate SEMA3B at the posttranscriptional level (58).

p53 is the first tumor suppressor gene to be discovered, which exerts its tumor suppressor effect mainly by inducing tumor cell apoptosis and inhibiting growth (59). In subsequent studies, more and more lncRNAs have been shown to be p53 effectors, and they are involved in tumor regulation in a p53-dependent manner (60). uc061hsf. 1 is a direct transcription target of p53. uc061hsf. 1 regulates the expression of the downstream transcription factor FoxA1 and inhibits the proliferation and migration of ESCC cells, indicating that uc061hsf.1 is a tumor suppressor LncRNA regulated by $\mathrm{P} 53$ (61).

\section{LONG-CHAIN NON-CODING RNAS CAN INDICATE THE PROGNOSIS OF PATIENTS WITH ESOPHAGEAL CANCER}

Studies have confirmed that the expression of LncRNAs, both carcinogenic and tumor suppressor LncRNAs, is associated with factors such as tumor volume, pathological stage, lymph node metastasis, and histological differentiation as well as overall survival (Table 2). The differential expression of these 
TABLE 2 | Common LncRNAs related to the pathology and prognosis of esophageal cancer.

\begin{tabular}{|c|c|c|c|c|c|c|}
\hline LncRNA & $\begin{array}{l}\text { Number of } \\
\text { samples }\end{array}$ & $\begin{array}{l}\text { Type of } \\
\text { samples }\end{array}$ & $\begin{array}{l}\text { Expression } \\
\text { level }\end{array}$ & Correlation & $\begin{array}{l}\text { Association with } \\
\text { survival }\end{array}$ & References \\
\hline \multirow[t]{2}{*}{ MALAT1 } & 100 cases & ESCC tumor tissues & Elevated & Related to TNM staging & Related to OS and DFS & $(67)$ \\
\hline & 320 cases & ESCC tumor tissues & Elevated & & $\begin{array}{l}\text { Independent predictor } \\
\text { of OS }\end{array}$ & $(62)$ \\
\hline CASC9 & 128 cases & ESCC tumor tissues & Elevated & $\begin{array}{l}\text { Related to tumor staging, lymph node metastasis, } \\
\text { and clinical staging }\end{array}$ & $\begin{array}{l}\text { Independent predictor of } \\
\text { OS and DFS }\end{array}$ & (63) \\
\hline DUXAP8 & 78 cases & ESCC tumor tissues & Elevated & Related to tumor staging and lymph node metastasis & Related to prognosis & $(41)$ \\
\hline \multirow[t]{2}{*}{ ZEB1-AS1 } & 26 cases & $\begin{array}{l}\text { Esophageal cancer } \\
\text { blood }\end{array}$ & Elevated & Related to the malignant progression of tumors & & (78) \\
\hline & 56 cases & ESCC tumor tissues & Elevated & Related to TNM staging and lymph node metastasis & Related to prognosis & $(64)$ \\
\hline \multirow[t]{2}{*}{$\mathrm{H} 19$} & 121 cases & ESCC tumor tissues & Elevated & Related to tumor volume and staging & Related to prognosis & (65) \\
\hline & $\begin{array}{l}\text { Public } \\
\text { database }\end{array}$ & ESCC tumor tissues & Elevated & & Related to prognosis & $(81)$ \\
\hline HNF1A-AS1 & 25 cases & EAC tumor tissues & Elevated & Related to the malignant progression of tumors & & $(20)$ \\
\hline BANCR & 142 cases & $\begin{array}{l}\text { ESCC tumor tissues } \\
\text { and blood }\end{array}$ & Elevated & $\begin{array}{l}\text { Related to histological grade, TNM staging, lymph } \\
\text { node metastasis }\end{array}$ & Related to OS & $(66)$ \\
\hline \multirow[t]{5}{*}{ UCA1 } & 100 cases & ESCC tumor tissues & Elevated & Related to TNM staging & Related to OS and DFS & $(67)$ \\
\hline & 70 cases & $\begin{array}{l}\text { Esophageal cancer } \\
\text { tissues }\end{array}$ & Elevated & Related to TNM staging and lymph node metastasis & & $(21)$ \\
\hline & 66 cases & $\begin{array}{l}\text { Esophageal cancer } \\
\text { tissues }\end{array}$ & Elevated & Related to TNM staging and tumor differentiation & Related to prognosis & $(22)$ \\
\hline & 110 cases & $\begin{array}{l}\text { Esophageal cancer } \\
\text { tissues }\end{array}$ & Elevated & $\begin{array}{l}\text { Related to TNM staging, lymph node metastasis, and } \\
\text { distant metastasis }\end{array}$ & Related to prognosis & (23) \\
\hline & 313 cases & $\begin{array}{l}\text { ESCC exosomes in } \\
\text { serum }\end{array}$ & Elevated & $\begin{array}{l}\text { Related to tumor staging, lymph node metastasis, } \\
\text { and clinical staging }\end{array}$ & Related to OS & $(70)$ \\
\hline \multirow[t]{2}{*}{ POU3F3 } & 313 cases & $\begin{array}{l}\text { ESCC exosomes in } \\
\text { serum }\end{array}$ & Elevated & $\begin{array}{l}\text { Related to tumor staging, lymph node metastasis, } \\
\text { and clinical staging }\end{array}$ & $\begin{array}{l}\text { Independent predictor } \\
\text { of OS }\end{array}$ & $(70)$ \\
\hline & 78 cases & $\begin{array}{l}\text { ESCC exosomes in } \\
\text { serum }\end{array}$ & Elevated & & Related to prognosis & $(86)$ \\
\hline CCAT2 & 33 cases & ESCC tumor tissues & Elevated & & Related to OS & (29) \\
\hline \multirow[t]{2}{*}{ HOTAIR } & 25 cases & ESCC tumor tissues & Elevated & Related to tumor differentiation & Related to prognosis & (38) \\
\hline & 100 cases & ESCC tumor tissues & Elevated & $\begin{array}{l}\text { Related to tumor differentiation, lymph node } \\
\text { metastasis, and clinical staging }\end{array}$ & $\begin{array}{l}\text { Independent predictor } \\
\text { of OS }\end{array}$ & $(68)$ \\
\hline uc002yug.2 & 684 cases & ESCC tumor tissues & Elevated & & Related to OS & (69) \\
\hline \multirow[t]{2}{*}{ PVT1 } & 156 cases & EAC tumor tissues & Elevated & $\begin{array}{l}\text { Related to histological grade and lymph node } \\
\text { metastasis }\end{array}$ & Related to prognosis & (26) \\
\hline & 76 cases & $\begin{array}{l}\text { ESCC tumor tissues } \\
\text { and serum }\end{array}$ & Elevated & $\begin{array}{l}\text { Related to TNM staging, lymph node metastasis, and } \\
\text { distant metastasis }\end{array}$ & Related to OS & $(27)$ \\
\hline \multirow[t]{2}{*}{ PCAT1 } & 130 cases & ESCC tumor tissues & Elevated & Related to lymph node metastasis and clinical staging & Related to prognosis & $(76)$ \\
\hline & 147 cases & ESCC serum & Elevated & Related to clinical staging & & $(77)$ \\
\hline \multirow[t]{3}{*}{ MEG3 } & 143 cases & ESCC tumor tissues & Reduced & $\begin{array}{l}\text { Related to TNM staging, depth of tumor invasion, } \\
\text { lymph node metastasis, and distant metastasis }\end{array}$ & $\begin{array}{l}\text { Independent predictor } \\
\text { of OS }\end{array}$ & $(51)$ \\
\hline & 28 cases & ESCC tumor tissues & Reduced & & Related to prognosis & $(52)$ \\
\hline & 43 cases & ESCC tumor tissues & Reduced & Related to TNM staging and lymph node metastasis & Related to prognosis & $(71)$ \\
\hline $\begin{array}{l}\text { SEMA3B- } \\
\text { AS1 }\end{array}$ & 138 cases & ESCC tumor tissues & Reduced & Related to TNM staging and lymph node metastasis & Related to prognosis & $(58)$ \\
\hline uc061hsf.1 & 34 cases & ESCC tumor tissues & Reduced & $\begin{array}{l}\text { Related to lymph node metastasis and tumor } \\
\text { differentiation }\end{array}$ & Related to prognosis & $(61)$ \\
\hline
\end{tabular}

OS, total survival; DFS, disease-free survival.

LncRNAs in tumor tissues can help evaluate tumor progression and prognosis in patients with esophageal cancer and provide new markers for further improving esophageal cancer treatment.

MALAT1 and CASC9 are highly expressed in ESCC tissues and are closely related to the TNM staging. They are important independent predictors of overall and disease-free survival. These LncRNAs can act as novel indicators of tumor progression and prognosis $(62,63)$. DUXAP8 and ZEB1-AS1 are also highly expressed in ESCC tissues. Analyses have found that their expression is positively correlated with ESCC state, lymph node metastasis, and 
prognosis and they are potential prognostic indicators for esophageal cancer $(41,64)$. Li et al. (65) found that $\mathrm{H} 19$ upregulation is associated with a large tumor size, high tumor stage, and short survival duration, indicating that $H 19$ expression could be a marker of malignant transformation and patient prognosis. Furthermore, there is a significant positive correlation between the expression of HNF1AAS1 and H19, suggesting that HNF1A-AS1 could also be a prognostic biomarker (20). Liu et al. (66) found that the expression of BANCR in the ESCC patients was related to histological grade, TNM stage, lymph node metastasis and OS. After the patient's tumor is removed, the expression level of $B A N C R$ returns to normal, and as the disease progresses, the expression level of $B A N C R$ gradually increases, which has certain potential for early diagnosis and evaluation of ESCC. Multiple studies have found that the expression of UCA1,POU3F3,CCAT2, HOTAIR, and uc002yug.2 is significantly higher in cancerous tissue than in normal adjacent tissues, and that high expression levels indicate shortened overall survival. This demonstrates that UCA1, POU3F3, CCAT2, HOTAIR, and uc002yug. 2 could be important prognostic factors among patients with esophageal cancer $(22,23,29,67-70)$.

In EAC, the correlation between LncRNA and prognosis is also shown. Xu et al. (26) compared EAC tissue with Barrett's esophagus and normal esophagus. The results showed that $P V T 1$ expression was up-regulated in EAC tissues, and PVT1 expression was related to histological grade, lymph node metastasis and survival.

Some other tumor suppressor LncRNAs can also act as novel prognostic indicators in esophageal cancer. MEG3 has obvious tumor suppressor properties in ESCC tissue, and its expression is negatively correlated with lymph node metastasis and TNM staging. Further studies have revealed that MEG3 expression is negatively correlated with the expression of its downstream target PSAT1, high levels of which are indicative of poor patient prognosis. This suggests that MEG3 is a potential prognostic marker that inhibits the EMT in tumor cells by inhibiting the Snail signaling pathway via PSAT1 (71). In line with this, Huang et al. (52) also proposed that MEG3 expression in ESCC tissues is related to tumor volume, lymph node metastasis, and pathological staging, and MEG3 is an independent predictor of disease-free and overall survival. The expressions of SEMA3BAS1 and uc061hsf.1 are reduced in ESCC tissues and have been found to be lower in esophageal cancer tissues with lymph node metastasis or poor differentiation. This suggests that the expression of SEMA3B-AS1 and uc061hsf.1 is closely related to prognosis among patients with esophageal cancer $(58,61)$.

\section{LIQUID BIOPSY LONG-CHAIN NON-CODING RNA IS CONDUCIVE TO THE EARLY DIAGNOSIS OF ESOPHAGEAL CANCER}

Even though ribonuclease levels in the blood are high, miRNA from tumor cells remains stable in serum and plasma. miRNA levels in the blood can be used as indicators of tumorigenesis. The US Food and Drug Administration has approved a series of miRNAs as tumor diagnostic indicators for clinical trials (72).
Similarly, LncRNA can also be detected in human blood. Therefore, the abnormal levels of LncRNA in the blood could be a potential indicator for the early diagnosis of esophageal cancer.

Multiple LncRNAs are also differentially expressed in serum in patients with cancer. Hu et al. (27) analyzed sera from 76 ESCC patients and found that serum PVT1 levels were related to lymph node metastasis, TNM staging, and postoperative metastasis. High levels of PVT1 often indicate a worse overall survival, suggesting that PVT1 could be used as a valuable serum marker for ESCC diagnosis and prognostication. BANCR is highly expressed in the plasma of ESCC patients. After tumor removal, plasma $B A N C R$ levels revert to the levels observed in healthy individuals. BANCR is closely related to the status of tumor activity, suggesting that it has certain potential for early diagnosis and evaluation of ESCC (66). The levels of GAS5 and $N E F$ in the cancer tissues and serum of ESCC patients are significantly lower than those in normal individuals, and the levels of GAS5 and NEF also decrease with an increase in tumor stage $(44,48)$.

An indicator for early tumor diagnosis should not only show significant differential expression but also be stable in the blood and be easy to detect. Tong et al. (73) tested the stability and diagnostic performance of circulating LncRNA-POU3F3 in the blood. They found that even after the serum undergoes multiple freeze-thaw cycles or is treated with acidic or alkaline solutions, POU3F3 can still be detected and remains stable. Through receiver operating characteristic curve analysis, they found that serum $P O U 3 F 3$ levels have good sensitivity and specificity in the prediction of ESCC and could be an ideal early diagnostic index for this type of malignancy.

Exosomes are the star molecules of tumor liquid biopsy. As a messenger of "intercellular communication," they can circulate in the whole body fluid, which makes it a new type of liquid biopsy marker to attract researchers' attention. Exosomes contain a large number of non-coding RNAs, such as microRNA (miRNA), cyclic RNA, and long-chain non-coding RNA (lncRNA). Especially during the development of tumors, exosomes can carry lncRNA that is more abundant than tumor cells. The lipid bilayer membrane structure of exosomes protects the non-coding RNA from being degraded, reduces the complexity of detecting multi-component body fluids, and increases the sensitivity and specificity of detection for low-abundance molecules (74). Therefore, LncRNA in exosomes can become an emerging biomarker.

Prostate cancer-related transcript 1 (PCAT1) was originally identified as an over-expressed lncRNA in prostate cancer by RNA sequencing, which can promote the progression of prostate cancer (75). In ESCC tissues, elevated PCAT1 expression is related to tumor lymph node metastasis and clinical staging (76). More importantly, PCAT1 was packaged into ESCC cellderived exosomes and highly expressed in serum, ultimately promoting tumor proliferation through ceRNA interaction with miR-326 (77). ZEB1-AS1 is derived from the ZEB1 promoter region. Exosomes have been detected in patients with esophageal cancer, and these patients have been found to show higher levels of ZEB1-AS1 than healthy individuals. 
Research has also found that ZEB1-AS1 can promote the proliferation of esophageal cancer cells by up-regulating the downstream effector protein ZEB1, demonstrating that ZEB1$A S 1$ can be found in peripheral blood exosomes and may be used as a new marker for early blood-based tumor detection (78). As the most potential indicator for diagnosing ESCC, the study found that $P O U 3 F 3$ also had persistently elevated expression in exosomes, and the expression level was not disturbed by experimental conditions (70).

\section{REGULATION OF LONG-CHAIN NON-CODING RNA EXPRESSION CAN ENHANCE TUMOR SENSITIVITY TO RADIOTHERAPY AND CHEMOTHERAPY}

Currently, esophageal cancer is typically treated with comprehensive strategies. Of these, neoadjuvant therapy combined with radiotherapy and chemotherapy can increase the surgical resection rate, reduce the risk of distant metastasis, and provide a therapeutic effect better than that of traditional surgical resection. However, LncRNAs affect not only the occurrence and development of esophageal cancer but also the sensitivity of tumor cells to radiotherapy and chemotherapy. Therefore, regulating the expression of LncRNAs in esophageal cancer tissues and reducing the resistance of tumor cells to radiotherapy and chemotherapy can be used as novel approaches to improve therapeutic efficacy and prevent tumor recurrence. Due to the large number of ESCC patients and the tendency to metastasize. Therefore, the current research focuses on LncRNA regulating the chemoresistance and radioresistance of ESCC.

Radiotherapy is an important step in the treatment of ESCC, and avoiding radiotherapy resistance of tumor tissue can effectively reduce recurrence and metastasis. MALAT1 is related to the radiotherapy sensitivity of tumors. Li et al. (79) overexpressed MALAT1 in ESCC cells and transplanted these cells to generate tumors in nude mice. After irradiation, they found that MALAT1 overexpression enhanced tumor cell viability and reduced apoptosis. This demonstrated that MALAT1 can inhibit the apoptosis induced by radiation and enhance the resistance of cells to radiotherapy, and MALAT1 silencing may enhance the sensitivity of cells to radiotherapy (80). Luo et al. (81) inhibited H19 in radiation-resistant esophageal cancer cells and found an increase in miR-22-3p expression, decrease in Wntl expression, and reduction in cell proliferation and migration. This suggested that $H 19$ can regulate the Wnt pathway via miR-22-3p and confer radiotherapy resistance. Therefore, knocking out $H 19$ and thereby enhancing the radiosensitivity of ESCC may be a new treatment strategy. Lin et al. (82) found that the expression of the tumor suppressor GAS5 in radiation-sensitive cells was higher than that in radiation-resistant cells. The overexpression of GAS5 can promote the reduction in miR-21 expression, increase the levels of RECK, and increase tumor cell apoptosis after radiotherapy. It has been suggested that GAS5 modulates miR-
21/RECK to increase the radiation sensitivity of tumor cells, and could therefore also serve as a target for improving the effect of radiotherapy.

ESCC is more sensitive to chemotherapy drugs, so chemotherapy also has good curative effect. Colon cancerrelated transcript 1 (CCAT1) was first discovered as a carcinogen in colon cancer (83). Inhibition of the CCAT1 gene was found to up-regulate the downstream target miR-143, reducing the expression of $\mathrm{Ki}-67$ and promoting G1 arrest. miR-143 targets the expression of PLK1 and BUBR1 in ESCC cells and promotes the sensitivity of tumor cells to cisplatin drugs (84). It has been suggested that CCAT1 can regulate the proliferation and chemotherapy resistance of esophageal cancer cells by regulating the miR-143/PLK1/BUBR1 axis. Prostate an-drogen regulated transcript 1 (PART1) is upregulated in gefitinib-resistant esophageal cancer cells and is associated with adverse effects of gefitinib treatment. The experimental knockout of PART1 can promote the death of gefitinib-resistant esophageal cancer cells and reduce the resistance of ESCC to gefitinib (85). POU3F3 is not only of great significance in the early diagnosis of ESCC, but also can guide the selection of chemotherapy drugs. Tumor cell-derived exosomes contain $P O U 3 F 3$, which induces the transformation of normal fibroblasts to tumor-associated fibroblasts, increases the level of IL-6 in the tumor microenvironment, and promotes cisplatin resistance in ESCC cells(86).

\section{APPLYING PUBLIC DATABASES TO DISCOVER MORE POTENTIALLY VALUABLE LONG-CHAIN NON-CODING RNAS}

The methods to study the regulatory function of LncRNA mainly include traditional biological experimental methods and modern computational methods of bioinformatics. Traditional biological experimental methods to identify the function of LncRNAs, although the results are accurate and reliable, have problems such as long experimental cycle time and high cost. With the continuous development of high-throughput sequencing technology, more and more LncRNAs have been discovered, and the functions of a large number of LncRNAs need to be clarified. Traditional biological experimental methods are obviously incompetent, and fast and efficient computational methods must be used to conduct data mining of the discovered relationship between LncRNAs and human diseases, and then infer the function of LncRNAs (87).

LncRNADisease: In 2012, Chen et al. (88) collected the relationship between LncRNAs in the regulation of human diseases reported in the PubMed database and developed the first database of LncRNAs in the regulation of human diseases. LncRNADisease gives the PubMed hyperlink of the original article for each LncRNA-disease association data, and annotates the detailed information of the LncRNA-disease association, including genomic information, sequence information, dysfunction type, etc. 
Lnc2Cancer: In 2015, Ning et al. (89) established an experimentally supported LncRNA database that specifically collects LncRNA-cancer associations. Each association data in the database includes the name of LncRNA and cancer, LncRNA sequence and location information, LncRNA expression pattern, experimental technique, LncRNA functional description, PubMed database hyperlink and other annotation information.

LncRNADisease2.0 is an updated version of LncRNADisease, which was established by Bao et al. (90) in 2018. Compared to the first version of LncRNADisease, LncRNADisease 2.0 has significant improvements. For example, transcriptional regulatory relationships between LncRNAs, mRNAs, and miRNAs are provided; disease names are mapped to the $\mathrm{MeSH}$ database, and quantitative confidence scores are provided for each LncRNA-disease association. LncRNADisease 2.0 is one of the most comprehensive databases for collecting LncRNA-disease association data.

\section{SUMMARY AND OUTLOOK}

As our understanding of LncRNA increases, our awareness of the key roles LncRNAs play in the functioning of various cells under normal and disease states is also increasing. LncRNAs participate in gene regulation via a variety of molecular mechanisms. For example, some LncRNAs act as a "molecular sponge" to regulate downstream miRNAs, whereas others act as epigenetic regulators that affect the expression of effector proteins or as protein chaperones to affect protein function. Studies have confirmed that there are several LncRNAs in tumor tissues and they perform various functions, and most studies have reported the specific molecular mechanisms underlying these processes. LncRNAs are promising new biomarkers that can assist in early disease diagnosis, improve the curative effect of treatment, and predict patient prognosis. Further, the discovered LncRNA regulatory networks and molecular pathways could provide potential avenues for targeted tumor therapy.

However, some LncRNAs participate in multiple molecular pathways in tumors. One example is HOTAIR, which is involved in both the miR-125/miR-143/HK2 axis and the miR-130a-5p/ ZEB1 pathway. It has been confirmed that signaling molecules

\section{REFERENCES}

1. Fan J, Liu Z, Mao X, Tong X, Zhang T, Suo C, et al. Global Trends in the Incidence and Mortality of Esophageal Cancer from 1990 to 2017. Cancer Med (2020) 9:e03338. doi:10.1002/cam4.3338

2. Bray F, Ferlay J, Soerjomataram I, Siegel RL, Torre LA, Jemal A. Global Cancer Statistics 2018: GLOBOCAN Estimates of Incidence and Mortality Worldwide for 36 Cancers in 185 Countries. CA: A Cancer J Clinicians (2018) 68(6): 394-424. doi:10.3322/caac. 21492

3. Wong MCS, Hamilton W, Whiteman DC, Jiang JY, Qiao Y, Fung FDH, et al. Global Incidence and Mortality of Oesophageal Cancer and Their Correlation with Socioeconomic Indicators Temporal Patterns and Trends in 41 Countries. Sci Rep (2018) 8(1):4522. doi:10.1038/s41598018-19819-8

4. Ohashi S, Miyamoto Si., Kikuchi O, Goto T, Amanuma Y, Muto M. Recent Advances from Basic and Clinical Studies of Esophageal Squamous Cell usually interact with each other and function together. A single LncRNA is not sufficient to drive cell signal transduction, and accordingly, a single signaling molecule may not work effectively. Therefore, our understanding of LncRNAs is still at the nascent stages. It is necessary to discover more LncRNAs related to signaling pathways, further characterize how LncRNAs and signaling molecules work co-operatively, and map the multifactor regulatory networks that include both LncRNA and miRNA. This will help in the application of LncRNAs as biomarkers for early tumor diagnosis and prognostic assessment and targets for precise treatment.

\section{AUTHOR CONTRIBUTIONS}

CY finished writing the article. KC provided supervision and final check.

\section{FUNDING}

This work was supported by grants from the National Natural Science Foundation of China (No. 81873455), National Natural Science Foundation of China Joint Fund Project (No. U1704173), and Henan Province Health Science and Technology Innovative Talents “51282” Project (Leading Talents) (2016).

\section{CONFLICT OF INTEREST}

The authors declare that the research was conducted in the absence of any commercial or financial relationships that could be construed as a potential conflict of interest.

\section{ACKNOWLEDGMENTS}

The authors would like to thank all the reviewers who participated in the review and MJ Editor (www.mjeditor.com) for its linguistic assistance during the preparation of this manuscript.

Carcinoma. Gastroenterology (2015) 149(7):1700-15. doi:10.1053/j.gastro. 2015.08.054

5. Maher B. ENCODE: The Human Encyclopaedia. Nature (2012) 489(7414): 46-8. doi:10.1038/489046a

6. Lee JT. Epigenetic Regulation by Long Noncoding RNAs. Science (2012) 338(6113):1435-9. doi:10.1126/science.1231776

7. Yoon J-H, Kim J, Gorospe M. Long Noncoding RNA Turnover. Biochimie (2015) 117:15-21. doi:10.1016/j.biochi.2015.03.001

8. Wang X, Sun W, Shen W, Xia M, Chen C, Xiang D, et al. Long Non-coding RNA DILC Regulates Liver Cancer Stem Cells via IL-6/STAT3 axis. J Hepatol (2016) 64(6):1283-94. doi:10.1016/j.jhep.2016.01.019

9. Zhou J, Yang L, Zhong T, Mueller M, Men Y, Zhang N, et al. H19 lncRNA Alters DNA Methylation Genome Wide by Regulating S-Adenosylhomocysteine Hydrolase. Nat Commun (2015) 6:10221. doi:10.1038/ncomms10221

10. Wu Y, Zhang L, Wang Y, Li H, Ren X, Wei F, et al. Long Noncoding RNA HOTAIR Involvement in Cancer. Tumor Biol (2014) 35(10):9531-8. doi:10. 1007/s13277-014-2523-7 
11. Yang F, Zhang L, Huo X-s., Yuan J-h., Xu D, Yuan S-x., et al. Long Noncoding RNA High Expression in Hepatocellular Carcinoma Facilitates Tumor Growth through Enhancer of Zeste Homolog 2 in Humans. Hepatology (2011) 54(5): 1679-89. doi:10.1002/hep.24563

12. Thin KZ, Liu X, Feng X, Raveendran S, Tu JC, LncRNA-Dancr. LncRNADANCR: A Valuable Cancer Related Long Non-coding RNA for Human Cancers. Pathol - Res Pract (2018) 214(6):801-5. doi:10.1016/j.prp.2018.04.003

13. Qi Y, Song C, Zhang J, Guo C, Yuan C. Oncogenic LncRNA CASC9 in Cancer Progression. Curr Pharm Des (2021) 27(4):575-82. doi:10.2174/ 1381612826666200917150130

14. Ji P, Diederichs S, Wang W, Böing S, Metzger R, Schneider PM, et al. MALAT1 , a Novel Noncoding RNA, and Thymosin $\beta 4$ Predict Metastasis and Survival in Early-Stage Non-small Cell Lung Cancer. Oncogene (2003) 22(39):8031-41. doi:10.1038/sj.onc. 1206928

15. Hu L, Wu Y, Tan D, Meng H, Wang K, Bai Y, et al. Up-regulation of Long Noncoding RNA MALAT1 Contributes to Proliferation and Metastasis in Esophageal Squamous Cell Carcinoma. J Exp Clin Cancer Res (2015) 34:7. doi:10.1186/s13046-015-0123-Z

16. Chen M, Xia Z, Chen $\mathrm{C}, \mathrm{Hu}$ W, Yuan Y. LncRNA MALAT1 Promotes Epithelial-To-Mesenchymal Transition of Esophageal Cancer through Ezh2-Notch1 Signaling Pathway. Anti-Cancer Drugs (2018) 29(8):767-73. doi:10.1097/cad.0000000000000645

17. Wang X, Li M, Wang Z, Han S, Tang X, Ge Y, et al. Silencing of Long Noncoding RNA MALAT1 by miR-101 and miR-217 Inhibits Proliferation, Migration, and Invasion of Esophageal Squamous Cell Carcinoma Cells. J Biol Chem (2015) 290(7):3925-35. doi:10.1074/jbc.m114.596866

18. Tan D, Wu Y, Hu L, He P, Xiong G, Bai Y, et al. Long Noncoding RNA H19 Is Up-Regulated in Esophageal Squamous Cell Carcinoma and Promotes Cell Proliferation and Metastasis. Dis Esophagus (2017) 30(1):1-9. doi:10.1111/ dote. 12481

19. Chen M-J, Deng J, Chen C, Hu W, Yuan Y-C, Xia Z-K. LncRNA H19 Promotes Epithelial Mesenchymal Transition and Metastasis of Esophageal Cancer via STAT3/EZH2 axis. Int J Biochem Cel Biol (2019) 113:27-36. doi:10.1016/j. biocel.2019.05.011

20. Yang X, Song JH, Cheng Y, Wu W, Bhagat T, Yu Y, et al. Long Non-coding RNAHNF1A-AS1regulates Proliferation and Migration in Oesophageal Adenocarcinoma Cells. Gut (2014) 63(6):881-90. doi:10.1136/gutjnl-2013305266

21. Wang P, Liu X, Han G, Dai S, Ni Q, Xiao S, et al. Downregulated lncRNA UCA1 Acts as ceRNA to Adsorb microRNA-498 to Repress Proliferation, Invasion and Epithelial Mesenchymal Transition of Esophageal Cancer Cells by Decreasing ZEB2 Expression. Cell Cycle (2019) 18(18):2359-76. doi:10. 1080/15384101.2019.1648959

22. Jiao C, Song Z, Chen J, Zhong J, Cai W, Tian S, et al. IncRNA-UCA1 Enhances Cell Proliferation through Functioning as a ceRNA of Sox4 in Esophageal Cancer. Oncol Rep (2016) 36(5):2960-6. doi:10.3892/or.2016.5121

23. Liu HE, Shi HH, Luo XJ. Upregulated Long Noncoding RNA UCA1 Enhances Warburg Effect via miR-203/HK2 Axis in Esophagal Cancer. J Oncol (2020) 2020:8847687. doi:10.1155/2020/8847687

24. Zhang W, Chen Q, Lei C. IncRNA MIAT Promotes Cell Invasion and Migration in Esophageal Cancer. Exp Ther Med (2020) 19(5):3267-74. doi:10.3892/etm.2020.8588

25. Zhang C, Xie L, Fu Y, Yang J, Cui Y. lncRNA MIAT Promotes Esophageal Squamous Cell Carcinoma Progression by Regulating miR-1301-3p/INCENP axis and Interacting with SOX2. J Cel Physiol (2020) 235(11):7933-44. doi:10. 1002/jcp. 29448

26. Xu Y, Li Y, Jin J, Han G, Sun C, Pizzi MP, et al. LncRNA PVT1 Up-Regulation Is a Poor Prognosticator and Serves as a Therapeutic Target in Esophageal Adenocarcinoma. Mol Cancer (2019) 18(1):141. doi:10.1186/s12943-0191064-5

27. $\mathrm{Hu}$ J, Gao W. Long Noncoding RNA PVT1 Promotes Tumour Progression via the miR-128/ZEB1 axis and Predicts Poor Prognosis in Esophageal Cancer. Clin Res Hepatol Gastroenterol (2021) 45(4):101701. doi:10.1016/j.clinre.2021. 101701

28. Kasagi Y, Oki E, Ando K, Ito S, Iguchi T, Sugiyama M, et al. The Expression of CCAT2, a Novel Long Noncoding RNA Transcript, and Rs6983267 SingleNucleotide Polymorphism Genotypes in Colorectal Cancers. Oncology (2017) 92(1):48-54. doi:10.1159/000452143
29. Yang C, Li F, Zhou W, Huang J. Knockdown of Long Non-coding RNA CCAT2 Suppresses Growth and Metastasis of Esophageal Squamous Cell Carcinoma by Inhibiting the $\beta$-catenin/WISP1 Signaling Pathway. J Int Med Res (2021) 49(5):3000605211019938. doi:10.1177/03000605211019938

30. Wang X, Wang X. Long Non-coding RNA colon Cancer-Associated Transcript 2 May Promote Esophageal Cancer Growth and Metastasis by Regulating the Wnt Signaling Pathway. Oncol Lett (2019) 18(2):1745-54. doi:10.3892/ol.2019.10488

31. Liang Y, Chen X, Wu Y, Li J, Zhang S, Wang K, et al. LncRNA CASC9 Promotes Esophageal Squamous Cell Carcinoma Metastasis through Upregulating LAMC2 Expression by Interacting with the CREB-Binding Protein. Cell Death Differ (2018) 25(11):1980-95. doi:10.1038/s41418-0180084-9

32. Wu Y, Hu L, Liang Y, Li J, Wang K, Chen X, et al. Up-regulation of lncRNA CASC9 Promotes Esophageal Squamous Cell Carcinoma Growth by Negatively Regulating PDCD4 Expression through EZH2. Mol Cancer (2017) 16(1):150. doi:10.1186/s12943-017-0715-7

33. Zhong $\mathrm{X}, \mathrm{Hu} \mathrm{X}$, Zhang L. Oncogenic Long Noncoding RNA FAL1 in Human Cancer. Mol Cell Oncol (2015) 2(2):e977154. doi:10.4161/23723556.2014. 977154

34. Liu T, Wang Z, Zhou R, Liang W. Focally Amplified lncRNA on Chromosome 1 Regulates Apoptosis of Esophageal Cancer Cells via DRP1 and Mitochondrial Dynamics. IUBMB Life (2019) 71(2):254-60. doi:10.1002/ iub. 1971

35. Liang XS, Sun Y, Liu T. Long Non-coding RNA FAL1 Regulated Cell Proliferation through Akt Pathway via Targeting PDK1 in Esophageal Cancer Cells. Eur Rev Med Pharmacol Sci (2018) 22(16):5214-22. doi:10. 26355/eurrev_201808_15719

36. Rinn JL, Kertesz M, Wang JK, Squazzo SL, Xu X, Brugmann SA, et al. Functional Demarcation of Active and Silent Chromatin Domains in Human HOX Loci by Noncoding RNAs. Cell (2007) 129(7):1311-23. doi:10.1016/j.cell.2007.05.022

37. Ma J, Fan Y, Feng T, Chen F, Xu Z, Li S, et al. HOTAIR Regulates HK2 Expression by Binding Endogenous miR-125 and miR-143 in Oesophageal Squamous Cell Carcinoma Progression. Oncotarget (2017) 8(49):86410-22. doi:10.18632/oncotarget.21195

38. Wang W, Wu D, He X, Hu X, Hu C, Shen Z, et al. CCL18-induced HOTAIR Upregulation Promotes Malignant Progression in Esophageal Squamous Cell Carcinoma through the miR-130a-5p-ZEB1 axis. Cancer Lett (2019) 460: 18-28. doi:10.1016/j.canlet.2019.06.009

39. Su W, Guo C, Wang L, Wang Z, Yang X, Niu F, et al. LncRNA MIR22HG Abrogation Inhibits Proliferation and Induces Apoptosis in Esophageal Adenocarcinoma Cells via Activation of the STAT3/c-Myc/FAK Signaling. Aging (2019) 11(13):4587-96. doi:10.18632/aging.102071

40. Li F-Z, Zang W-Q. Knockdown of lncRNAXLOC_001659 Inhibits Proliferation and Invasion of Esophageal Squamous Cell Carcinoma Cells. World J Gastroenterol (2019) 25(42):6299-310. doi:10.3748/wjg. v25.i42.6299

41. Xu LJ, Yu XJ, Wei B, Hui HX, Sun Y, Dai J, et al. Long Non-coding RNA DUXAP8 Regulates Proliferation and Invasion of Esophageal Squamous Cell Cancer. Eur Rev Med Pharmacol Sci (2018) 22(9):2646-52. doi:10.26355/ eurrev_201805_14959

42. Wang Y, Zhang W, Liu W, Huang L, Wang Y, Li D, et al. Long Noncoding RNA VESTAR Regulates Lymphangiogenesis and Lymph Node Metastasis of Esophageal Squamous Cell Carcinoma by Enhancing VEGFC mRNA Stability. Cancer Res (2021) 81(12):3187-99. doi:10.1158/0008-5472.can-20-1713

43. Ma C, Shi X, Zhu Q, Li Q, Liu Y, Yao Y, et al. The Growth Arrest-specific Transcript 5 (GAS5): a Pivotal Tumor Suppressor Long Noncoding RNA in Human Cancers. Tumor Biol (2016) 37(2):1437-44. doi:10.1007/s13277-0154521-9

44. Wang G, Sun J, Zhao H, Li H. Long Non-coding RNA (lncRNA) Growth Arrest Specific 5 (GAS5) Suppresses Esophageal Squamous Cell Carcinoma Cell Proliferation and Migration by Inactivating Phosphatidylinositol 3-kinase (PI3K)/AKT/Mammalian Target of Rapamycin (mTOR) Signaling Pathway. Med Sci Monit (2018) 24:7689-96. doi:10.12659/msm.910867

45. Huang J, Li Y, Lu Z, Che Y, Sun S, Mao S, et al. Long Non-coding RNA GAS5 Is Induced by Interferons and Plays an Antitumor Role in Esophageal Squamous Cell Carcinoma. Cancer Med (2018) 7:3157-67. doi:10.1002/cam4.1524 
46. Wang K, Li J, Xiong G, He G, Guan X, Yang K, et al. Negative Regulation of IncRNA GAS5 by miR-196a Inhibits Esophageal Squamous Cell Carcinoma Growth. Biochem Biophysical Res Commun (2018) 495(1):1151-7. doi:10. 1016/j.bbrc.2017.11.119

47. Liang W-C, Ren J-L, Wong C-W, Chan S-O, Waye MM-Y, Fu W-M, et al. LncRNA-NEF Antagonized Epithelial to Mesenchymal Transition and Cancer Metastasis via Cis-Regulating FOXA2 and Inactivating Wnt $/ \beta$-Catenin Signaling. Oncogene (2018) 37(11):1445-56. doi:10.1038/s41388-017-0041-y

48. Zhang J, Hu SL, Qiao CH, Ye JF, Li M, Ma HM, et al. LncRNA-NEF Inhibits Proliferation, Migration and Invasion of Esophageal Squamous-Cell Carcinoma Cells by Inactivating Wnt/ß-Catenin Pathway. Eur Rev Med Pharmacol Sci (2018) 22(20):6824-31. doi:10.26355/eurrev_201810_16150

49. Modarresi F, Faghihi MA, Lopez-Toledano MA, Fatemi RP, Magistri M, Brothers SP, et al. Inhibition of Natural Antisense Transcripts In Vivo Results in Gene-specific Transcriptional Upregulation. Nat Biotechnol (2012) 30(5):453-9. doi:10.1038/nbt.2158

50. Zhao H, Diao C, Wang X, Xie Y, Liu Y, Gao X, et al. LncRNA BDNF-AS Inhibits Proliferation, Migration, Invasion and EMT in Oesophageal Cancer Cells by Targeting miR-214. J Cel Mol Med (2018) 22:3729-39. doi:10.1111/ jcmm. 13558

51. Dong Z, Zhang A, Liu S, Lu F, Guo Y, Zhang G, et al. Aberrant MethylationMediated Silencing of lncRNA MEG3 Functions as a ceRNA in Esophageal Cancer. Mol Cancer Res (2017) 15(7):800-10. doi:10.1158/1541-7786.mcr-160385

52. Huang Z-L, Chen R-P, Zhou X-T, Zhan H-L, Hu M-M, Liu B, et al. Long Noncoding RNA MEG3 Induces Cell Apoptosis in Esophageal Cancer through Endoplasmic Reticulum Stress. Oncol Rep (2017) 37(5):3093-9. doi:10.3892/ or.2017.5568

53. Yao J, Zhou B, Zhang J, Geng P, Liu K, Zhu Y, et al. A New Tumor Suppressor LncRNA ADAMTS9-AS2 Is Regulated by DNMT1 and Inhibits Migration of Glioma Cells. Tumor Biol (2014) 35(8):7935-44. doi:10.1007/s13277-0141949-2

54. Liu D, Wu K, Yang Y, Zhu D, Zhang C, Zhao S. Long Noncoding RNA ADAMTS9-AS2 Suppresses the Progression of Esophageal Cancer by Mediating CDH3 Promoter Methylation. Mol Carcinog (2020) 59(1):32-44. doi: $10.1002 / \mathrm{mc} .23126$

55. Dong Z, Li S, Wu X, Niu Y, Liang X, Yang L, et al. Aberrant HypermethylationMediated Downregulation of Antisense lncRNA ZNF667-AS1 and its Sense Gene ZNF667 Correlate with Progression and Prognosis of Esophageal Squamous Cell Carcinoma. Cell Death Dis (2019) 10(12):930. doi:10.1038/ s41419-019-2171-3

56. Guo W, Liu S, Dong Z, Guo Y, Ding C, Shen S, et al. Aberrant MethylationMediated Silencing of lncRNA CTC-276P9.1 Is Associated with Malignant Progression of Esophageal Squamous Cell Carcinoma. Clin Exp Metastasis (2018) 35(1-2):53-68. doi:10.1007/s10585-018-9881-2

57. Zhao B, Cao P, Hu S, Li F, Kong K, Zu Y. LncRNA-NBAT-1 Modulates Esophageal Cancer Proliferation via PKM2. Am J Transl Res (2019) 11(9): 5978-87.

58. Dong Z, Liang $\mathrm{X}, \mathrm{Wu} \mathrm{X}$, Kang $\mathrm{X}$, Guo $\mathrm{Y}$, Shen $\mathrm{S}$, et al. Promoter Hypermethylation-Mediated Downregulation of Tumor Suppressor Gene SEMA3B and IncRNA SEMA3B-AS1 Correlates with Progression and Prognosis of Esophageal Squamous Cell Carcinoma. Clin Exp Metastasis (2019) 36(3):225-41. doi:10.1007/s10585-019-09964-3

59. Bieging KT, Mello SS, Attardi LD. Unravelling Mechanisms of P53-Mediated Tumour Suppression. Nat Rev Cancer (2014) 14(5):359-70. doi:10.1038/ $\operatorname{nrc} 3711$

60. Zhang A, Xu M, Mo Y-Y. Role of the IncRNA-P53 Regulatory Network in Cancer. J Mol Cel Biol (2014) 6(3):181-91. doi:10.1093/jmcb/mju013

61. Yao J, Zhang H, Li H, Qian R, Liu P, Huang J. P53-regulated IncRNA uc061hsf.1 Inhibits Cell Proliferation and Metastasis in Human Esophageal Squamous Cell Cancer. IUBMB Life (2020) 72(3):401-12. doi:10.1002/iub. 2196

62. Wang C, Zhang Q, Hu Y, Zhu J, Yang J. Emerging Role of Long Non-coding RNA MALAT1 in Predicting Clinical Outcomes of Patients with Digestive System Malignancies: A Meta-Analysis. Oncol Lett (2019) 17(2):2159-70. doi:10.3892/ol.2018.9875

63. Gao GD, Liu XY, Lin Y, Liu HF, Zhang GJ. LncRNA CASC9 Promotes Tumorigenesis by Affecting EMT and Predicts Poor Prognosis in Esophageal
Squamous Cell Cancer. Eur Rev Med Pharmacol Sci (2018) 22(2):422-9. doi:10. 26355/eurrev_201801_14191

64. Zhao Y, Wang N, Zhang X, Liu H, Yang S. LncRNA ZEB1-AS1 DownRegulation Suppresses the Proliferation and Invasion by Inhibiting ZEB1 Expression in Oesophageal Squamous Cell Carcinoma. J Cel Mol Med (2019) 23(12):8206-18. doi:10.1111/jcmm.14692

65. Li X, Yang H, Wang J, Li X, Fan Z, Zhao J, et al. High Level of lncRNA H19 Expression Is Associated with Shorter Survival in Esophageal Squamous Cell Cancer Patients. Pathol - Res Pract (2019) 215(11):152638. doi:10.1016/j.prp. 2019.152638

66. Liu Z, Yang T, Xu Z, Cao X. Upregulation of the Long Non-coding RNA BANCR Correlates with Tumor Progression and Poor Prognosis in Esophageal Squamous Cell Carcinoma. Biomed Pharmacother (2016) 82:406-12. doi:10. 1016/j.biopha.2016.05.014

67. Kang K, Huang YH, Li HP, Guo SM. Expression of UCA1 and MALAT1 LongChain Non-coding RNAs in Esophageal Squamous Cell Carcinoma Tissues Is Predictive of Patient Prognosis. Arch Med Sci (2018) 14(4):752-9. doi:10.5114/ aoms.2018.73713

68. Li X, Wu Z, Mei Q, Li X, Guo M, Fu X, et al. Long Non-coding RNA HOTAIR, a Driver of Malignancy, Predicts Negative Prognosis and Exhibits Oncogenic Activity in Oesophageal Squamous Cell Carcinoma. Br J Cancer (2013) 109(8): 2266-78. doi:10.1038/bjc.2013.548

69. Wu H, Zheng J, Deng J, Zhang L, Li N, Li W, et al. LincRNA-uc002yug.2 Involves in Alternative Splicing of RUNX1 and Serves as a Predictor for Esophageal Cancer and Prognosis. Oncogene (2015) 34(36):4723-34. doi:10. 1038/onc.2014.400

70. Yan S, Du L, Jiang X, Duan W, Li J, Xie Y, et al. Evaluation of Serum Exosomal lncRNAs as Diagnostic and Prognostic Biomarkers for Esophageal Squamous Cell Carcinoma. Cancer Manag Res (2020) 12:9753-63. doi:10.2147/cmar. s250971

71. Li M, Liu L, Zhang W, Zhan H, Chen R, Feng J, et al. Long Non-coding RNA MEG3 Suppresses Epithelial-To-Mesenchymal Transition by Inhibiting the PSAT1-dependent GSK-33/Snail Signaling Pathway in Esophageal Squamous Cell Carcinoma. Oncol Rep (2020) 44(5):2130-42. doi:10.3892/or.2020.7754

72. Zhang J, Zhao H, Gao Y, Zhang W. Secretory miRNAs as Novel Cancer Biomarkers. Biochim Biophys Acta (Bba) - Rev Cancer (2012) 1826(1):32-43. doi:10.1016/j.bbcan.2012.03.001

73. Tong Y-S, Wang X-W, Zhou X-L, Liu Z-H, Yang T-X, Shi W-H, et al. Identification of the Long Non-coding RNA POU3F3 in Plasma as a Novel Biomarker for Diagnosis of Esophageal Squamous Cell Carcinoma. Mol Cancer (2015) 14:3. doi:10.1186/1476-4598-14-3

74. Shao H, Im H, Castro CM, Breakefield X, Weissleder R, Lee H. New Technologies for Analysis of Extracellular Vesicles. Chem Rev (2018) 118(4):1917-50. doi:10.1021/acs.chemrev.7b00534

75. Prensner JR, Iyer MK, Balbin OA, Dhanasekaran SM, Cao Q, Brenner JC, et al. Transcriptome Sequencing across a Prostate Cancer Cohort Identifies PCAT1 , an Unannotated lincRNA Implicated in Disease Progression. Nat Biotechnol (2011) 29(8):742-9. doi:10.1038/nbt.1914

76. Shi W-h., Wu Q-q., Li S-q., Yang T-x., Liu Z-h., Tong Y-s., et al. Upregulation of the Long Noncoding RNA PCAT-1 Correlates with Advanced Clinical Stage and Poor Prognosis in Esophageal Squamous Carcinoma. Tumor Biol (2015) 36(4):2501-7. doi:10.1007/s13277-014-2863-3

77. Huang L, Wang Y, Chen J, Wang Y, Zhao Y, Wang Y, et al. Long Noncoding RNA PCAT1, a Novel Serum-Based Biomarker, Enhances Cell Growth by Sponging miR-326 in Oesophageal Squamous Cell Carcinoma. Cel Death Dis (2019) 10(7):513. doi:10.1038/s41419-019-1745-4

78. Zhang YG, Zhou MW, Bai L, Han RY, Lv K, Wang Z. Extracellular Vesicles Promote Esophageal Cancer Progression by Delivering lncZEB1-AS1 between Cells. Eur Rev Med Pharmacol Sci (2018) 22(9):2662-70. doi:10.26355/ eurrev_201805_14962

79. Li Z, Zhou Y, Tu B, Bu Y, Liu A, Kong J. Long Noncoding RNA MALAT1 Affects the Efficacy of Radiotherapy for Esophageal Squamous Cell Carcinoma by Regulating Cks1 Expression. J Oral Pathol Med (2017) 46(8):583-90. doi:10. 1111/jop. 12538

80. Farooqi A, Legaki E, Gazouli M, Rinaldi S, Berardi R. MALAT1 as a Versatile Regulator of Cancer: Overview of the Updates from Predatory Role as Competitive Endogenous RNA to Mechanistic Insights. Curr Cancer Drug Targets (2020) 21:192-202. doi:10.2174/1568009620999200730183110 
81. Luo W, Liu W, Yao J, Zhu W, Zhang H, Sheng Q, et al. Downregulation of H19 Decreases the Radioresistance in Esophageal Squamous Cell Carcinoma Cells. OncoTargets Ther (2019) 12:4779-88. doi:10.2147/ott.s203235

82. Lin J, Liu Z, Liao S, Li E, Wu X, Zeng W. Elevation of Long Non-coding RNA GAS5 and Knockdown of microRNA-21 Up-Regulate RECK Expression to Enhance Esophageal Squamous Cell Carcinoma Cell Radio-Sensitivity after Radiotherapy. Genomics (2020) 112(3):2173-85. doi:10.1016/j.ygeno.2019. 12.013

83. Nissan A, Stojadinovic A, Mitrani-Rosenbaum S, Halle D, Grinbaum R, Roistacher M, et al. Colon Cancer Associated Transcript-1: a Novel RNA Expressed in Malignant and Pre-malignant Human Tissues. Int J Cancer (2012) 130(7):1598-606. doi:10.1002/ijc.26170

84. Hu M, Zhang Q, Tian XH, Wang JL, Niu YX, Li G. IncRNA CCAT1 Is a Biomarker for the Proliferation and Drug Resistance of Esophageal Cancer via the miR-143/PLK1/BUBR1 axis. Mol Carcinog (2019) 58(12):2207-17. doi:10. 1002/mc.23109

85. Kang M, Ren M, Li Y, Fu Y, Deng M, Li C. Exosome-mediated Transfer of lncRNA PART1 Induces Gefitinib Resistance in Esophageal Squamous Cell Carcinoma via Functioning as a Competing Endogenous RNA. J Exp Clin Cancer Res (2018) 37(1):171. doi:10.1186/s13046-018-0845-9

86. Tong Y, Yang L, Yu C, Zhu W, Zhou X, Xiong Y, et al. Tumor-Secreted Exosomal lncRNA POU3F3 Promotes Cisplatin Resistance in ESCC by
Inducing Fibroblast Differentiation into CAFs. Mol Ther - Oncolytics (2020) 18:1-13. doi:10.1016/j.omto.2020.05.014

87. Moran VA, Perera RJ, Khalil AM. Emerging Functional and Mechanistic Paradigms of Mammalian Long Non-coding RNAs. Nucleic Acids Res (2012) 40(14):6391-400. doi:10.1093/nar/gks296

88. Chen G, Wang Z, Wang D, Qiu C, Liu M, Chen X, et al. LncRNADisease: a Database for Long-Non-Coding RNA-Associated Diseases. Nucleic Acids Res (2013) 41(Database issue):D983-6. doi:10.1093/nar/gks1099

89. Ning S, Zhang J, Wang P, Zhi H, Wang J, Liu Y, et al. Lnc2Cancer: a Manually Curated Database of Experimentally Supported lncRNAs Associated with Various Human Cancers. Nucleic Acids Res (2016) 44(D1):D980-D985. doi:10.1093/nar/gkv1094

90. Bao Z, Yang Z, Huang Z, Zhou Y, Cui Q, Dong D. LncRNADisease 2.0: an Updated Database of Long Non-coding RNA-Associated Diseases. Nucleic Acids Res (2019) 47(D1):D1034-d1037. doi:10.1093/nar/gky905

Copyright (c) 2022 Yang and Chen. This is an open-access article distributed under the terms of the Creative Commons Attribution License (CC BY). The use, distribution or reproduction in other forums is permitted, provided the original author(s) and the copyright owner(s) are credited and that the original publication in this journal is cited, in accordance with accepted academic practice. No use, distribution or reproduction is permitted which does not comply with these terms. 